\title{
Le théâtre du corps. Pour une définition du terme physical theatre
}

Demis Quadri

\section{(2) OpenEdition \\ 12 Journals}

Édition électronique

URL : https://journals.openedition.org/edl/3228

DOI : $10.4000 /$ edl.3228

ISSN : 2296-5084

Éditeur

Université de Lausanne

\section{Édition imprimée}

Date de publication : 24 septembre 2020

Pagination : 249-266

ISBN : 978-2-940331-74-1

ISSN : 0014-2026

\section{Référence électronique}

Demis Quadri, « Le théâtre du corps. Pour une définition du terme physical theatre », Études de lettres [En ligne], 313 | 2020, mis en ligne le 24 septembre 2021, consulté le 25 septembre 2021. URL : http:// journals.openedition.org/edl/3228; DOI : https://doi.org/10.4000/edl.3228 


\section{LE THÉÂTRE DU CORPS. POUR UNE DÉFINITION DU TERME PHYSICAL THEATRE}

Cette contribution tente une définition ouverte du terme physical theatre. En mettant en évidence un certain nombre de thèmes - mouvement, formation, travail collaboratif, tensions conceptuelles, dramaturgie d'acteur, horizontalité, etc. - soulevés par les chercheurs et les artistes qui ont abordé ce sujet, et en tenant compte des Six Viewpoints développés par Mary Overlie - espace, forme, temps, émotion, mouvement et histoire -, elle propose de traiter cette forme de théâtre comme phénomène émergent d'un réseau (network) constitué de ces éléments en interaction réciproque. Au lieu de définir le physical theatre comme opposé au théâtre "littéraire», il sera caractérisé par le positionnement conscient de l'acteur sur un certain nombre de dimensions (textualitécorporalité, représentation-performance, dramatique-postdramatique, etc.).

\section{Un problème initial: la conception du théâtre}

La tentative de définir le physical theatre - qui, en français, peut être traduit par "théâtre du corps» - est motivée par l'idée qu'une forme de théâtre très physique - comportant des aspects proches de la danse et même du sport - pourra profiter de repères théoriques et d'outils conceptuels permettant de la décrire en profondeur et de la rendre lisible. Malgré l'hybridation des genres artistiques et performatifs des dernières années qui, dans un sens positif, a permis de faire tomber de nombreuses barrières entre disciplines, les idées des professionnels, des pédagogues, des étudiants, des théoriciens et des spectateurs concernant le théâtre n'ont pas toujours suivi l'évolution des faits. Ceci entraîne le risque, d'une part, de favoriser des lectures partielles et inadéquates des productions théâtrales et, d'autre part, de diluer les approches théoriques suite 
à la négligence de certains contextes, formes et contenus. La recherche d'une définition du théâtre physique ne vise pas à créer de nouvelles cages et à élever de nouveaux murs, mais à encourager une discussion qui valorise les atouts de la diversité.

Avant de discuter certains éléments qui peuvent guider l'explication du terme physical theatre, il faut constater que le concept de théâtre luimême est controversé, et que ces controverses ont des répercussions pour ce projet. En essayant de saisir ce qu'est le théâtre tout court, il est utile de se demander, en reprenant une suggestion du théâtrologue italien Luigi Allegri - inspirée par le Manifesto per un nuovo teatro de Pier Paolo Pasolini -, quelle est l'idée de théâtre la plus communément partagée ${ }^{1}$. Cette approche est également valable lorsque nous cherchons à définir un phénomène théâtral comme le physical theatre qui s'écarte de cette même idée partagée, parce que cela nous permet d'en examiner les différences et leurs conséquences.

Une grande partie de la recherche sur le théâtre publiée dans le monde occidental est évidemment orientée vers l'étude des pratiques scéniques en Europe et en Amérique du Nord; l'idée du théâtre que se fait une grande partie du public (surtout les non-spécialistes) est, elle aussi, fortement influencée par les principales traditions des scènes européennes et nord-américaines. Or, dans sa tentative d'identifier les principaux débats sur la théorie du théâtre dans différentes parties du monde et de les combiner avec les discussions sur des questions clés sur le plan théorique, Glenn Odom attire l'attention sur certaines différences entre les textes fondateurs des traditions japonaise et européenne, en l'occurrence les traités de Zeami Motokiyo et La Poétique d'Aristote ${ }^{2}$. Zeami imagine son théâtre comme une production en direct avec des acteurs jouant devant des spectateurs. Aristote, par contre, se concentre sur les éléments techniques de l'écriture dramatique, avec beaucoup moins d'attention à la mise en scène et aux spécificités des arts vivants. Cette divergence entre deux œuvres fondamentales de l'esthétique théâtrale ${ }^{3}$ montre la tendance de l'Occident à comprendre, sous l'influence des philosophes

I. Cf. L. Allegri, Prima lezione sul teatro, p. 9-31.

2. Odom le fait à partir des réflexions de Thomas Rimer, célèbre expert américain et traducteur de la littérature et du théâtre japonais. Cf. M. Zeami, On the Art of No Drama.

3. Le cas japonais n'en est qu'un parmi d'autres que l'on peut citer en considérant par exemple d'autres ouvrages fondamentaux comme le Nätyaśāstra indien ou le Yueji 
grecs, le théâtre à partir du texte et, s'il y a spectacle, à privilégier la parole au détriment d'autres éléments comme le chant ou les mouvements des corps ${ }^{4}$, tandis que la tradition théâtrale japonaise porte son attention sur la dimension performative et les conditions de production ${ }^{5}$.

Dans son livre très influent sur le théâtre postdramatique, le chercheur allemand Hans-Thies Lehmann explore les tendances des arts de la scène contemporains qui conçoivent le théâtre comme une affirmation d'expérience directe et sans médiation, au lieu de le voir comme une médiation entre texte et spectateurs. Ce qui intéresse Lehmann, c'est un théâtre qui est:

[...] plus présence que représentation, plus expérience partagée que communication, plus processus que produit, plus manifestation que signification, plus impulsion d'énergie qu'information ${ }^{6}$.

Malgré l'influence des réflexions de Lehmann et, surtout, malgré les innombrables exemples de pratique théâtrale à la base de ses positions, l'idée de théâtre semble toujours centrée sur la logique du mot et sur une approche qui est au moins partiellement aristotélicienne, ce qu'illustrent également des célèbres manuels de dramaturgie tels que ceux d'Yves Lavandier ${ }^{7}$ et de José Luis Alonso De Santos ${ }^{8}$. Cette situation crée des difficultés pour des formes de théâtre du corps qui, d'une part, diffèrent des formes auxquelles le grand public et les experts sont plus habitués et qui, d'autre part, cherchent à trouver leur place parmi d'autres arts de la scène comme la danse, le cirque contemporain, la danse-théâtre, la performance, etc.

chinois. Cf. G. Ottaviani, I fondamenti del teatro in Asia; F. Dupont, Aristote ou le vampire du théâtre occidental.

4. Cf. Aristote, Poétique 6, 1449a38-b20, qui établit une hiérarchie claire entre: 1. Intrigue (muthos) 2. Caractère (èthos) 3. Pensée (dianoia) 4. Expression (lexis) 5. Composition de chant (melopoiia) et 6. Spectacle (opsis).

5. G. Odom, World Theories of Theatre, p. 25.

6. H.-T. Lehmann, Postdramatisches Theater, p. 146.

7. Y. Lavandier, La dramaturgie.

8. J. L. Alonso De Santos, Manual de teoría y práctica teatral. 
Un corpus de définitions du physical theatre

The Routledge Companion to Theatre and Performance de Paul Allain et Jen Harvie contient un très bon résumé des lignes qui peuvent guider la réflexion sur le physical theatre. Selon eux, il s'agit en premier lieu d'un terme qui, bien que largement utilisé, est problématique, car caractérisé par une histoire incertaine, différente selon les lieux et les périodes de référence ${ }^{9}$. Cependant, quelques publications que je présenterai brièvement dans cette section mettent en évidence des éléments clés du physical theatre. Parmi celles-ci, il y a l'article d'Allain et Harvie, car malgré le fait qu'il n'ajoute pas d'observations nouvelles, il a le mérite d'arriver à synthétiser et à problématiser les éléments centraux de la question ${ }^{10}$. Une grande qualité du Routledge Companion to Theatre and Performance, qui donne toujours des suggestions bibliographiques pertinentes, est en outre de proposer des chapitres consacrés à des concepts fondamentaux pour le théâtre du corps, comme ceux sur le mouvement, la performativité et l'entraînement ${ }^{11}$. D'autres portent sur des protagonistes des arts de la scène qui ont contribué à en définir les contours, comme Eugenio Barba, Pina Bausch, Jerzy Grotowsky et Jacques Lecoq ${ }^{12}$ ou bien sur des créations qui ont conduit à l'émergence de ce phénomène, comme Dead Dreams of Monochrome Men de DV8 Physical Theatre ${ }^{13}$.

Pour la recherche sur le théâtre du corps, sont fondamentaux les deux volumes publiés par John Keefe et Simon Murray sous le titre commun de Physical Theatres: ils prennent en considération un réseau complexe de thèmes et de textes utiles en traversant des aspects liés à la genèse, aux contextes, à la terminologie, aux pratiques passées et présentes, aux formes de préparation et de formation et à des questions fondamentales liées à la politique, à la culture, à la physicalité, etc. En même temps, ils

9. P. Allain, J. Harvie, The Routledge Companion to Theatre and Performance, p. 225. Cf. D. Quadri, «Le théâtre en mouvement».

Io. P. Allain, J. Harvie, The Routledge Companion to Theatre and Performance, p. 225$s q$.

II. Ibid., p. 209 sq., 222 sq. et 256-258.

I2. Ibid., p. 25-27, 27-29, 54-56 et 60 sq.

13. Ibid., p. 110 sq. 
évitent pertinemment d'enfermer l'idée du théâtre du corps dans une forme unitaire de théâtre - d'où le pluriel dans le titre ${ }^{14}$.

Une troisième source est Through the Body de Dymphna Callery: il s'agit d'un guide pratique sur le théâtre du corps qui se présente comme une collection de possibilités d'exploration et d'exercices dans les domaines du devising ${ }^{15}$, du travail avec les masques, du jeu, de l'improvisation, etc. dans un esprit de théâtre total ${ }^{16}$. Through the Body est un livre qui, en plus de fournir d'autres composantes au puzzle du physical theatre, montre explicitement - en tant qu'outil de travail pratique - l'importance d'une approche fondée dans la pratique, lorsqu'il s'agit de comprendre le fonctionnement d'un théâtre qui repose principalement sur l'utilisation du corps et du mouvement, sans être colonisé par le langage verbal. Un complément utile au livre de Callery est The Body Speaks de Lorna Marshall qui, en proposant aussi un bon nombre d'exercices, illustre les besoins - pour le travail des artistes - de la prise de conscience de leur propre corps et de l'expansion des possibilités expressives par l'utilisation du mouvement comme instrument de communication artistique $^{17}$.

Dans les paragraphes suivants, j'essaierai de prendre en considération ces textes afin d'esquisser une première définition (ouverte) du terme physical theatre. Dans un second temps, j'essaierai de fournir quelques éléments supplémentaires pour rendre la définition à la fois plus flexible et plus précise.

I4. J. Keefe, S. Murray, Physical Theatres. A Critical Raeder; S. Murray, J. Keefe, Physical Theatres. A Critical Introduction.

15. Le devising est une méthode de création et de réalisation scénique qui se passe souvent d'un texte préexistant et qui implique la participation collaborative de l'ensemble du groupe d'artistes dans tous les aspects et phases de la mise en scène. Cf. P. Allain, J. Harvie, The Routledge Companion to Theatre and Performance, p. 172. L'expression française la plus proche de devised theatre est "écriture de plateau", où le terme "écriture», pris au sens métaphorique, implique aussi une création collaborative.

I6. D. Callery, Through the Body.

17. L. Marshall, The Body Speaks. 


\section{Une première approche}

Bien qu'ils ne lient pas explicitement leurs pages sur le thème du mouvement à une réflexion sur le physical theatre, Allain et Harvie l'abordent en prenant en considération des noms comme ceux de Pina Bausch et de Jacques Lecoq, et des concepts comme «entraînement» et "performativité", déjà mentionnés plus haut: les deux chercheurs établissent ainsi un réseau de relations entre ces concepts et les approches de travail scénique où ceux-ci sont particulièrement importants. Le mouvement est certes une composante intrinsèque de tout événement théâtral ou performatif dont une définition exhaustive se doit de rendre compte ${ }^{18}$. Ce qui change dans le cas spécifique des théâtres du corps, c'est l'accent mis sur le mouvement comme noyau de l'expression et de la communication, une caractéristique beaucoup moins prise en compte dans la perception des traditions théâtrales «littéraires». Pour une définition du physical theatre, il est donc moins important d'opérer avec des approches opposées du théâtre que de mettre en évidence les différentes pondérations et ingrédients dans un continuum. Le poids que le mouvement peut prendre dans les théâtres du corps a un lien direct avec l'entraînement physique (ou psychophysique) de l'acteur, le training. Cet entrầnement contribue non seulement à la préparation du performeur, mais a aussi une influence importante sur la dramaturgie et sur la création théâtrale. Cette idée d'entraînement a des racines lointaines, par exemple dans les expériences de pédagogues paradigmatiques du théâtre «littéraire» comme Stanislavski. Ce dernier, cependant, considérait cet aspect du travail de l'acteur comme un outil permettant de le mettre à la disposition du personnage et du texte de l'auteur ${ }^{19}$, et moins comme un élément qui contribue à la construction de structures, de formes et de contenus théâtraux à travers les recherches du performeur.

Une source plus appropriée est la tradition des formes d'entraînement développées dans une lignée de danseurs-acteurs qui a traversé l'histoire des arts de la scène à partir au moins de Jean-Georges Noverre, jusqu'à des artistes tels que Pina Bausch ${ }^{20}$. Selon Allain et Harvie, un objectif

I8. P. Allain, J. Harvie, The Routledge Companion to Theatre and Performance, p. 209 sq.

19. M. De Marinis, Il teatro dopo l'età d'oro, p. 111-118.

20. Cf. C. Lo Iacono, Il danzatore attore da Noverre a Pina Bausch. 
fondamental de l'entraînement des acteurs est le développement de certaines compétences qui améliorent, dans des conditions d'exécution variables, la gestion de l'imprévisibilité des réactions du public, l'élaboration de l'intensité des performances dans la perspective de l'acteur et le partage de l'expérience entre les artistes et les spectateurs. Dans le cadre de son entraînement, l'acteur s'exerce en intégrant le corps, la voix et l'esprit, travaillant ainsi la possibilité de laisser l'impulsion conduire l'action, sans que le jeu soit pris en défaut par l'auto-jugement ou la réflexion intellectuelle ${ }^{21}$. Plus loin, Allain et Harvie traitent d'un autre objectif de l'entraînement, à savoir aller au-delà des habitudes individuelles ou communes des acteurs. Il en résulte un genre de réceptivité que le public perçoit dans le travail et dans les dynamiques d'un groupe d'artistes qui réussissent à manifester une qualité particulière d'ensemble ${ }^{22}$. En ce sens, l'entraînement des acteurs affecte les processus de création du physical theatre comme théâtre centré - en termes grotowskiens - sur "ce qui se passe entre spectateur et acteur ${ }^{23}$. Du point de vue d'une dramaturgie traditionnelle, les influences de l'entraînement se matérialisent en particulier par la parenté de celui-ci avec l'improvisation théâtrale ${ }^{24}$.

L'entraînement de l'acteur du théâtre physique joue également un rôle important dans Physical Theatres. A Critical Introduction, où le thème a droit à un chapitre entier ${ }^{25}$. Une autre contribution importante de cette étude est l'identification de quelques tensions essentielles pour comprendre certains développements marquant l'histoire contemporaine du théâtre occidental. Il s'agit notamment des tensions entre théâtre dramatique et postdramatique, théâtre et performance, approche par tâche (task) et approche plus psychologisante, ou encore entre présence et représentation. Particulièrement pertinente pour le physical theatre est la tension entre l'acting et le performing, impliquant celle entre le personnage et la "performance persona". Très souvent, on constate la tendance, dans les théâtres du corps, à se distancier d'un acting, d'une interprétation dans le sens de la représentation d'un personnage. L'accent est alors mis sur l'idée d'un acteur qui se présente comme «interprète de soi-même» (avec

2I. Ibid., p. 256.

22. Ibid., p. 257.

23. J. Grotowski, Vers un théâtre pauvre, p. 31.

24. Cf. P. Allain, J. Harvie, The Routledge Companion to Theatre and Performance, p. 256.

25. Cf. S. Murray, J. Keefe, Physical Theatres. A Critical Introduction, p. 117 sq. 
les difficultés que le terme «soi-même» comporte). Cette vision se poursuit par l'effort de supprimer, par l'utilisation de tâches à exécuter, tout ce qui pourrait assumer un rôle médiateur entre acteurs et public, bref par la tentative de rejeter le «spectre» de l'illusion théâtrale ${ }^{26}$. Murray et Keefe mettent bien en évidence la nature problématique d'une mise en œuvre qui se prétend basée sur une physicalité non médiée par des constructions culturelles ou linguistiques. Ce qui devient central pour le théâtre du corps est précisément le positionnement variable sur la ligne de tension entre personnage et acteur/performeur ${ }^{27}$. Cette tension ouvre un espace qui peut mener à des formes scéniques plus déterminées, qui ne sont plus ni danse, ni théâtre de prose. La même ouverture s'opère autour de la tension entre approche dramatique et postdramatique. Ce qui donne sens à une dramaturgie du physical theatre est justement de renouer avec les origines étymologiques du drame: avec une signification qui n'est pas seulement d' "action", mais d'"acte chargé de conséquences» - comme c'était le cas chez Eschyle ${ }^{28}$-, avant que le mot ne se spécialise au sens de «drame» ou de "tragédie» ${ }^{29}$.

On revient ainsi à une concaténation cause-effet qui ne conduit pas nécessairement à une dramaturgie traditionnelle, tout en se rattachant néanmoins à des formes de "tissage" dramaturgique que l'on peut qualifier de théâtrales. La référence à l'idée de tissage favorise un lien avec celle de texte au sens étymologique de "tissu» ${ }^{30}$, ce qui permet de comparer les éléments qui s'entrecroisent dans la construction d'une pièce basée sur un texte écrit avec ceux qui conduisent à une création de physical theatre - où ce ne sont pas les entrelacements des mots et des phrases qui sont dramaturgiquement centraux, mais ceux des corps et des mouvements dans l'espace et le temps de la scène.

26. Ibid., p. 20-22.

27. Cet aspect est bien illustré par le travail de création de Dead Dreams of Monochrome Men du DV8 Physical Theatre, où l'histoire d'un personnage, tirée de reportages sur des événements réels, est entrelacée avec les expériences, les réflexions et les physicalités des danseurs/acteurs qui ont donné vie à la pièce. Cf. P. Allain, J. Harvie, The Routledge Companion to Theatre and Performance, p. 110 sq.

28. Cf. Eschyle, Agamemnon, 533, à propos du crime de Pâris.

29. P. Chantraine, Dictionnaire étymologique de la langue grecque, p. 284.

30. Cf. <www.cnrtl.fr/etymologie/texte> [20 avril 2020] et E. Barba, N. Savarese, L'arte segreta dell'attore, p. 46-51. 
Dymphna Callery soutient que le théâtre du corps, en raison de ses déclinaisons très différentes, ne peut pas être codifié, mais qu'on trouve dans ses manifestations des parallèles avec les concepts d'artisanat, de travail et de jeu collectifs, et de découverte. Les parallèles identifiés par Callery concernent l'accent mis sur l'artiste-créateur plutôt que sur l'artiste-interprète, les processus de travail collaboratifs, les pratiques enracinées dans le travail sur les corps, l'ouverture de la relation entre la scène et le public, et le caractère central de l'aspect «en direct» de l'art du théâtre ${ }^{31}$. Il est indéniable qu'un artiste-interprète a aussi une dimension créatrice dans son travail qui, cependant, est plus orientée vers sa propre contribution à la lecture scénique d'un personnage et d'une histoire définie par un auteur, que vers la construction d'une dramaturgie originale basée sur des contenus et des formes proposés par les acteurs eux-mêmes. En ce qui concerne la question des processus de travail collaboratifs, il est intéressant de considérer également certaines réflexions de Lorna Marshall. Dans son livre The Body Speaks, Marshall souligne que l'utilisation du langage implique toujours la présence d'un "autre» et que, pour travailler, l'acteur a besoin de la présence de cet «autre» pour trouver sa propre impulsion à communiquer et pour découvrir comment celle-ci prend forme ${ }^{32}$. Marshall parle ensuite de la relation de l'acteur avec le texte d'un auteur de théâtre, mais les réflexions sur l'«autre» prennent une valeur particulière si l'on considère ces théâtres du corps où le centre du processus créatif passe par la collaboration entre les acteurs et, donc, par les interactions entre un certain nombre de "soi» et un certain nombre d' «autres». Le théâtre physique tend à horizontaliser les relations entre les différents artistes impliqués, même lorsque, en fin de compte, les décisions finales sont prises par un metteur en scène ou par un chorégraphe à la vision duquel le travail des performeurs peut être soumis sans que leur contribution à la dimension dramaturgique et structurelle de la production théâtrale ne perde sa centralité. Dans son livre La drammaturgia da Diderot à Beckett, Luigi Allegri, au cours d'une réflexion sur l'après-Deuxième Guerre mondiale, écrit qu'après la parenthèse d'autonomie de la scène dans la période de l'avant-garde artistique du début du XXe siècle - du Futurisme au Dada -, s'est opérée une réappropriation du pouvoir de la part des dramaturges et des

31. D. Callery, Through the Body, p. 5.

32. L. Marshall, The Body Speaks, p. 170. 
auteurs, une sorte de revanche de l'«opérateur littéraire» sur l'«opérateur théâtral» (metteur en scène, acteur, etc.) ${ }^{33}$. À propos des relations de pouvoir entre les différentes personnes impliquées dans une production théâtrale, le philosophe et sémioticien italien Ferruccio Rossi-Landi parle même, à travers une analogie entre le texte écrit et le capital (qui dans cette perspective seraient les deux a priori par rapport à la production du spectacle et des marchandises), d'une approche "capitaliste» au théâtre. Selon lui, l'écrivain de théâtre peut, dans certains cas, être associé à la classe dominante en matière de droit perçu à utiliser de manière instrumentale le travail des "classes subalternes» dans la construction du spectacle théâtral ${ }^{34}$. Une manifestation exemplaire de ce phénomène est la prolifération des didascalies et des indications de mise en scène dans les textes d'écrivains comme Samuel Beckett, qui essaient de pré-écrire la mise en scène en préfigurant le décor, les mouvements des acteurs, les intonations, les gestes, les expressions, etc., en demandant fidélité au monde et aux intentions de l'auteur ${ }^{35}$. Une telle prise de contrôle est plus difficile dans une dramaturgie telle que celle des théâtres du corps, où le type de collaboration demandé par la création théâtrale exige des relations plus horizontales, déjà implicites dans la focalisation sur la relation acteur-spectateur mentionnée plus haut. Une tendance à l'horizontalité se manifeste aussi dans l'utilisation des ressources d'un théâtre qui tend à transcender les frontières entre disciplines ${ }^{36}$ : pour sortir des logiques organisationnelles du langage écrit et du parcours qui mène depuis l'auteur/émetteur, via l'acteur/canal, au public/récepteur, une création basée sur le corps et le mouvement implique un point de vue non hiérarchique. Elle permet, d'une part, une évolution qui passe par la succession d'une multitude de hiérarchies nées et dissoutes au cours du travail et, d'autre part, une réelle confrontation avec la complexité des situations des acteurs dans les processus interconnectés d'entraînement et de création ${ }^{37}$.

33. L. Allegri, La drammaturgia da Diderot à Beckett, p. 156.

34. F. Rossi-Landi, Semiotica e ideologia, p. 50.

35. L. Allegri, La drammaturgia da Diderot à Beckett, p. 156 sq.

36. P. Allain, J. Harvie, The Routledge Companion to Theatre and Performance, p. 225.

37. M. Overlie, Standing in Space, p. 79. 
La plupart des éléments considérés dans les paragraphes précédents sont retenus par la définition suivante qui caractérise le physical theatre comme:

[...] une forme de théâtre où le corps et le mouvement de l'acteur sont au centre du processus dramaturgique[: 1$]$ e corps [y] est un instrument de travail, le mouvement un moyen d'expression et une source de formes et de contenus artistiques ${ }^{38}$.

Cette définition permet de prendre en compte la diversité des phénomènes définis comme physical theatre. Elle a l'avantage, par rapport à d'autres définitions ouvertes des théâtres du corps, de mettre en évidence une approche compositionnelle différente de celles des formes de théâtre de prose qui, par ailleurs, peuvent être basées sur un texte écrit mais recourir à une forme de jeu très corporelle. Cette dernière approche n'est pas nécessairement en contradiction avec une logique enracinée dans des structures verbales: la logique qui anime le développement des scènes peut dériver de l'enchaînement d'un scénario écrit, et non pas de la dynamique entre les physicalités des interprètes, même lorsque, par exemple, une pièce comme Arlequin valet de deux maîtres (Il servitore di due padroni) de Carlo Goldoni est mise en scène dans la tradition de la commedia dell'arte, avec un accompagnement important de gestes et mouvements.

Certains aspects apparemment absents de cette définition, tels que ceux liés à une dramaturgie théâtrale et à la relation acteur-spectateur, sont pour leur part implicites dans le mot "théâtre». En pensant à ce dernier, il est intéressant de revenir à une perspective étymologique: le mot «théâtre» vient - à travers le latin ${ }^{39}$ - d'un mot grec qui signifie «le lieu où l'on regarde» et qui à son tour se lie au mot dont dérive "théorie»; des termes donc qui nous font penser à l'action de voir, mais aussi à celles de concevoir, de comprendre, d'entendre ${ }^{40}$. Cette approche étymologique nous permet de légitimer l'idée d'un théâtre qui naît du corps et du mouvement des acteurs, en développant des contenus qui ne se

38. Accademia Teatro Dimitri, p. 24. Cf. Y. Schmidt, S. Marinucci, S. Bocchini, D. Quadri, A. Rey, «DisAbility on Stage», p. 77.

39. Cf. <www.cnrtl.fr/etymologie/théâtre> [20 avril 2020].

40. P. Chantraine, Dictionnaire étymologique de la langue grecque, p. 408 sq. 
basent pas de manière substantielle sur des mécanismes linguistiques et qui n'atteignent pas le public essentiellement par les canaux de la parole.

\section{Une modification avec Mary Overlie}

Cette définition de physical theatre permet cependant des lectures très différentes. Elle laisse planer de nombreux doutes, à commencer par ceux qui concernent la difficulté de définir le théâtre même et donc de se référer à sa définition. C'est pourquoi je voudrais proposer deux modifications : élargir la définition en m'inspirant de la théorie des réseaux et y associer les Six Viewpoints développés par Mary Overlie ${ }^{41}$.

Un réseau est un ensemble d'éléments reliés par des interactions qui donnent lieu, à partir de leur comportement collectif, à des phénomènes émergents qui ne peuvent pas être prévus à partir des éléments isolés ${ }^{42}$. L'avantage de voir les théâtres du corps comme des phénomènes émergents d'un réseau d'éléments réside dans le fait, d'une part, de prendre en compte une grande variété d'approches différentes du théâtre - mais avec des caractéristiques communes importantes - et d'autre part, de rendre la définition suffisamment flexible pour que le système ne s'effondre pas si l'on y ajoute ou enlève des éléments. Cette approche rappelle aussi un principe du travail de formation de l'acteur développé par l'acteur, metteur en scène et pédagogue russo-américain Michail Tchekhov, et qui a rappelé par son élève Mala Powers qu'un acteur doit maîtriser simultanément une certaine quantité d'éléments: imagination, travail d'ensemble, sens de la forme, sens de la globalité, irradiation, composition, tâche, point focal, style, etc. Si leur nombre est très élevé, l'artiste de la scène bien formé peut être rassuré par le fait que le réseau composé par ces éléments fonctionne par une suite de réactions qui s'active d'elle-même à partir d'une attention consciente à seulement un nombre limité de ceux$\mathrm{ci}^{43}$. La théorie des réseaux se prête à la lecture d'un écrit très orienté vers la pratique comme celui de Tchekhov, ce qui suggère que cette approche se traduit bien dans une perspective qui ne se limite pas à un regard théorique. Il y a des différences substantielles entre l'approche de

4I. Cf. M. Overlie, Standing in Space.

42. G. Caldarelli, M. Catanzaro, Networks, p. 1-6.

43. M. Čechov, La tecnica dell'attore, p. 7-12. 
Tchekhov et la théorie des réseaux: dans le premier cas, on travaille sur des matériaux déjà connus qui permettent logiquement à une série d'éléments de s'éveiller les uns les autres; alors que, dans le second, émerge un phénomène nouveau. Mais ce qui importe est le point de comparaison: les deux approches contiennent un moment de découverte dans la production artistique. Une perspective qui ne se limite pas à un regard théorique est de toute façon nécessaire pour essayer de comprendre un domaine, celui du physical theatre, sans le plier aux logiques séquentielles, lexicales et grammaticales du langage écrit ou parlé.

Pour préciser la définition du théâtre du corps comme phénomène émergent d'un réseau d'éléments, il me semble utile de l'articuler avec les Six Viewpoints de Mary Overlie qui propose six éléments: espace (space), forme (shape), temps (time), émotion (emotion), mouvement (movement) et histoire (story) ${ }^{44}$. Certains de ces "points de vue» reprennent des éléments déjà abordés précédemment, mais pour une approche basée sur l'idée de réseau, il est important qu'ils soient également mis en évidence comme formant ses nœuds. Les points de vue identifiés par Mary Overlie ne sont d'ailleurs absolument pas le domaine exclusif du théâtre du corps: ce que je voudrais cependant souligner c'est que, dans cette forme de théâtre, ils ont une place centrale spécifique à tous les niveaux des processus de création et de mise en scène. Pour décrire et comprendre le physical theatre de manière satisfaisante, il vaut mieux mettre en évidence - comme mentionné précédemment - sa position flexible dans un continuum qui inclut, dans ce cas, théâtre «littéraire», danse, cirque contemporain, danse-théâtre, performance, mime corporel, etc. Ces arts du spectacle ont entre eux des frontières, mais aussi des caractéristiques communes de sorte que ces différences ne peuvent pas toujours être définies et séparées de manière claire et satisfaisante.

Notre culture repose souvent sur une pensée d'opposition ${ }^{45}$, mais il est plus pertinent, pour une compréhension du théâtre du corps, d'adopter une perspective de complémentarité qui permet de prendre en compte les relations entre corps, esprit et émotions. Le réseau d'éléments proposé par Mary Overlie doit, lui aussi, être considéré en termes de complémentarité et, du moins dans le contexte d'une définition générale

44. M. Overlie, Standing in Space, p. 5-13, 15-19, 21-28, 29-34, 35-41, 43-51.

45. Cf. P. Odifreddi, Il museo dei numeri, p. 91. 
de physical theatre, d'horizontalité ${ }^{46}$. Dans ce cadre, je voudrais souligner le rôle du viewpoint "histoire» (story), qui permet de mettre en évidence un aspect du théâtre du corps qui, autrement, risque d'être négligé. Dans son travail, Mary Overlie conçoit l'«histoire» comme la «logique spécifique qui fonctionne comme organisation de séquences d'informations ${ }^{47}$. Elle aurait en fait préféré utiliser le terme «logique»(logic), mais comme elle travaillait sur ce viewpoint à l'époque du Minimalisme Conceptuel, quand des artistes, comme Merce Cunningham et John Cage, pour sensibiliser le public à une sphère qui allait au-delà du récit, proposaient un rejet total du mot "histoire», elle a choisi de maintenir ce terme: les histoires sont très importantes pour la communication et l'expérience humaines, et ne peuvent pas être mises de côté à la légère ${ }^{48}$. Je pense que l'idée d'histoire proposée par Overlie peut aussi s'appliquer au physical theatre et que l'importance d'utiliser ce terme est également valable dans ce cas: pour qu'il s'agisse de théâtre, et qu'il soit perçu comme tel, il est nécessaire que le physical theatre raconte des histoires, aussi déstructurées, tordues, minimalistes, paradoxales, renversées, bouleversées ou liées à des logiques non linguistiques soient-elles. L'idée d'histoire nous ramène aussi à la question de départ concernant le physical theatre ou le théâtre du corps: pour comprendre de quoi il s'agit, plutôt que de l'encadrer dans une définition fermée, il vaut mieux rester ouvert face aux histoires qui découlent des différentes interactions possibles entre les composantes mises en évidence dans ces pages.

\section{Conclusion ouverte}

Les branches de l'histoire des arts de la scène qui ont conduit au développement du physical theatre ${ }^{49}$ ont réuni un certain nombre d'éléments dans la création théâtrale: l'entraînement et la physicalité des acteurs, la centralité du mouvement, un fort degré de performativité, etc. En même temps, elles ont donné naissance à des esthétiques et à des techniques

46. Cf. M. Overlie, Standing in Space, p. 79-86.

47. Ibid., p. 43.

48. Ibid., p. 45 sq.

49. Pour approfondir le sujet, cf. F. Cruciani, Registi pedagoghi e comunità teatrali nel Novecento; M. De Marinis, Mimo e teatro nel Novecento; Id., In cerca dell'attore. 
théâtrales qui sont difficiles à enfermer dans une définition. L'idée même d'une définition unique et fermée risque d'ailleurs de conduire à une contradiction avec l'hypothèse qui considère le physical theatre comme une forme de théâtre qui évite la colonisation par les règles de la langue et de la littérature. Pour comprendre les logiques du corps et du mouvement qui façonnent le physical theatre, mieux vaut thématiser le holisme et la complémentarité du système corps-esprit-émotion, que les critères d'exclusion qui caractérisent les définitions - comme par exemple la distinction binaire entre tragédie et comédie. Pour tenter une médiation entre les besoins spécifiques d'une définition et les spécificités du théâtre du corps, il parait utile de partir d'un concept tel que celui de la théorie des réseaux pour rendre compte de la complexité, de la variété et de la logique de cette approche de la création scénique. Par rapport aux questions soulevées par les chercheurs et les artistes considérées dans cet article, il est toutefois utile d'élargir le discours et d'apporter quelques précisions sous la forme d'une grille de concepts telle que celle proposée par Mary Overlie avec ses Six Viewpoints. De cette façon, on obtient une définition ouverte, non réductible à quelques phrases, mais composée d'un réseau d'éléments qui peuvent être présents en quantités et qualités différentes, peut-être pas toujours tous ensemble, et qui dans leurs interactions donnent lieu à des performances en cohérence avec les perspectives du physical theatre explicitées ici.

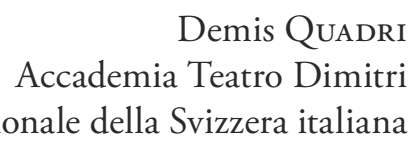

Scuola universitaria professionale della Svizzera italiana 


\section{BIBLIOGRAPHIE}

Accademia Teatro Dimitri. Physical Theatre. Bachelor of Arts in Theatre. Master of Arts in Theatre. Master of Arts in Artistic Research, Lugano, La Buona Stampa, 2018.

Alonso De Santos, José Luis, Manual de teoría y práctica teatral, Barcelona, Castalia, 2007.

Allain, Paul, Harvie, Jen, The Routledge Companion to Theatre and Performance, Routledge, London/New York, 2014 (2006).

Allegri, Luigi, La drammaturgia da Diderot à Beckett, Roma/Bari, Laterza, 2008.

—, Prima lezione sul teatro, Roma/Bari, Laterza, 2012.

Aristote, Poétique, traduction Barbara Gernez, Paris, Les Belles Lettres, 2008 (2001).

Barba, Eugenio, Savarese, Nicola, L'arte segreta dell'attore. Un dizionario di antropologia teatrale, Bari, Edizioni di Pagina, 2011.

Caldarelli, Guido, Catanzaro, Michele, Networks. A Very Short Introduction, Oxford, Oxford University Press, 2012.

Callery, Dymphna, Through the Body. A Practical Guide to Physical Theatre, New York, Routledge / London, Nick Hern Books, 2001. Čechov, Michail, La tecnica dell'attore, a cura di Mala Powers, Roma, Dino Audino, 2001.

Chantraine, Pierre, Dictionnaire étymologique de la langue grecque.

Histoire des mots, Paris, Klincksieck, 2009.

Cruciani, Fabrizio, Registi pedagoghi e comunità teatrali nel Novecento, Roma, Editori \& Associati, 1995.

De Marinis, Marco, Mimo e teatro nel Novecento, Firenze, La Casa Usher, 1993.

-, In cerca dell'attore. Un bilancio del Novecento teatrale, Roma, Bulzoni, 2000.

—, Il teatro dopo l'età d'oro. Novecento e oltre, Roma, Bulzoni, 2013.

Dupont, Florence, Aristote ou le vampire du théâtre occidental, Paris, Aubier, 2007.

Grotowski, Jerzy, Vers un théâtre pauvre, Lausanne, La Cité, 1971. 
Keefe, John, Murray, Simon, Physical Theatres. A Critical Reader, London/New York, Routledge, 2007.

-, Physical Theatres. A Critical Introduction, London/New York, Routledge, 2007.

Lavandier, Yves, La dramaturgie. L'art du récit, Bruxelles, Les Impressions nouvelles, 2019 ( $8^{\mathrm{e}}$ édition).

Lemmann, Hans-Thies, Postdramatisches Theater, Frankfurt am Main, Verlag der Autoren, 1999.

Lo Iacono, Concetta (a cura di), Il danzatore attore da Noverre a Pina Bausch, Roma, Dino Audino, 2007.

Marshall, Lorna, The Body Speaks. Performance and Physical Expression, London/Oxford/NewYork/New Delhi/Sydney, Bloomsbury, 2008.

Murray, Simon, Keefe, John, Physical Theatres. A Critical Introduction, London/New York, Routledge, 2007.

Odifreddi, Piergiorgio, Il museo dei numeri. Da zero verso l'infinito, storie dal mondo della matematica, Milano, Rizzoli, 2014.

ODom, Glenn, World Theories of Theatre, London/New York, Routledge, 2017.

Ottaviani, Gioia, I fondamenti del teatro in Asia. Nātyaśāstra. Yueji. I Trattati di Zeami, Roma, Bulzoni, 2007.

Overlie, Mary, Standing in Space: The Six Viewpoints. Theory \& Practice, Billings, Artcraft Printers, 2016.

QuAdri, Demis, «Le théâtre en mouvement. La recherche sur le physical theatre passe par la Suisse italienne", in Alain Clavien, Claude Hauser, François Vallotton (dir.), Théatre et scènes politiques, Lausanne, Antipodes, 2014, p. 201-210.

Rossi-Landi, Ferruccio, Semiotica e ideologia, Milano, Bompiani, 1972.

Schmidt, Yvonne, Marinucci, Sarah, Bocchini, Sara, Quadri, Demis, Rey, Anton, "DisAbility on Stage - Exploring the Physical in Dance and Performer Training", Synnyt/Origins: Finnish Studies in Art Education, 2 (2018), p. 74-87.

Zeami, Motokiyo, On the Art of No Drama, traduit par Thomas Rimer, Princeton, Princeton Library of Asian Translations, 1984. 
